\title{
A COMBINATORIAL ANALOG OF LYAPUNOV'S THEOREM FOR INFINITESIMALLY GENERATED ATOMIC VECTOR MEASURES
}

\author{
PETER A. LOEB ${ }^{1}$
}

\begin{abstract}
It is shown that the range of a measure obtained by the addition of infinitesimal vectors is convex up to infinitesimal errors.
\end{abstract}

By a well-known theorem of Lyapunov, the range in $R^{n}$ of a nonatomic totally finite vector-measure is convex (see [5]). Recent developments in economics (see [3]) have established the need for a similar theorem for a purely atomic measure on a *finite set $A$, where the measure of each atom $a \in A$ is a vector of infinitesimal length. The desired result is a corollary of the following theorem of Steinitz ([7, pp. 167-172]; also see [1, pp. 148-149]): Given any finite collection of vectors in $n$-space $R^{n}$ with sum 0 and maximum norm $M$, there is an ordering $v_{1}, v_{2}, \cdots, v_{p}$ of those vectors such that the norm of any partial sum $\sum_{i=1}^{j} v_{i}$ is smaller than $2 n M$. (Note that the norm in Steinitz's theorem need not be the Euclidean norm, and if it is, $2 n M$ may not be the best constant for dimension $n$.) (See [2] and [4].)

Let ${ }^{*} R$ and ${ }^{*} N$ denote the nonstandard models for the real numbers $R$ and natural numbers $N$ in a fixed enlargement of a structure that contains $R$. (See [6].) We write $a \simeq b$ when $a \in{ }^{*} R, b \in{ }^{*} R$ and $a-b$ is infinitesimal, and we denote by $|v|$ the Euclidean distance of any vector $v$ from the origin 0. Fix $n \in{ }^{*} N, n$ finite or infinite. If $u$ and $v$ are vectors in ${ }^{*} R^{n}$, we shall write $u \simeq v$ if $|u-v| \simeq 0$. Recall that a *finite set is a set for which there is an internal one-to-one correspondence with an initial segment of $* N$; such a set has all the "formal" properties of a finite set.

THEOREM. Let $A$ be $a{ }^{*}$ finite set, and for each $a \in A$, let $v(a)$ be $a$ vector in $n$-space ${ }^{*} R^{n}$ with $n v(a) \simeq 0$. For each internal set $B \subset A$, set

Received by the editors April 3, 1972 and, in revised form, November 17, 1972.

AMS (MOS) subject classifications (1970). Primary 26A98, 28A45, 46G10.

Key words and phrases. Reordered series, partial sums, Lyapunov theorem, nonstandard analysis, infinitesimal atomic vector measures, convex range.

${ }^{1}$ This work was supported by NSF Grant GP 14785 and a grant from the University of Illinois Center for Advanced Study. The author is indebted to Professors S. Kakutani and Donald J. Brown for several helpful conversations.

(C) American Mathematical Society 1973 
$S(B)=\sum_{a \in B} v(a)$. Then the following is true:

(i) If $S(A) \not 0$, there is an ordering $v_{1}, v_{2}, \cdots, v_{p}$ of the set $\{v(a): a \in A\}$ so that, for each $j \in *^{*} N$ with $1 \leqq j \leqq p, \sum_{i=1}^{j} v_{i} \simeq \lambda S(A)$ where $\lambda \in{ }^{*} R$.

(ii) Given internal sets $B \subset A$ and $C \subset A$ and given $\lambda \in{ }^{*} R$ with $0<\lambda<1$, there is an internal set $D \subset A$ with $S(D) \simeq \lambda S(B)+(1-\lambda) S(C)$.

Proof. To prove (i), let $P_{L}$ denote the projection of ${ }^{*} R^{n}$ onto the line $L$ through 0 and $S(A)$, and let $P_{H}$ denote the projection of ${ }^{*} R^{n}$ onto the hyperplane $H$ perpendicular to $L$ at 0 . The sum of the vectors $\left\{P_{H}(v(a)): a \in A\right\}$ is 0 . Therefore by Steinitz's theorem, there is an ordering $v_{1}, v_{2}, \cdots, v_{p}$ of the set $\{v(a): a \in A\}$ so that, for any $j \in *^{*} N$ with $1 \leqq j \leqq p$,

$$
\left|P_{H} \sum_{i=1}^{j} v_{i}\right|=\left|\sum_{i=1}^{j} P_{H}\left(v_{i}\right)\right| \leqq 2 n \max _{a_{\Xi} A}|v(a)| \simeq 0,
$$

whence $\sum_{i=1}^{j} v_{i} \simeq P_{L} \sum_{i=1}^{j} v_{i}=\lambda S(A)$ for some $\lambda \in *^{*} R$. This proves (i) and more, for given $\lambda \in{ }^{*} R$ with $0<\lambda<1$, there is a first $\omega \in{ }^{*} N$ such that $\left|\sum_{i=1}^{\omega} P_{L}\left(v_{i}\right)\right| \geqq \lambda|S(A)|$, and $\sum_{i=1}^{\omega} P_{L}\left(v_{i}\right)$ and $S(A)$ are on the same side of $H$. There is thus an $\omega \in^{*} N$ for which $\sum_{i=1}^{\omega} P_{L}\left(v_{i}\right) \simeq \lambda S(A)$. We now obtain statement (ii) from this fact by replacing $A$ with $B-C$ and $C-B$ and noting that, for $0<\lambda<1$,

$$
\lambda S(B)+(1-\lambda) S(C)=\lambda S(B-C)+(1-\lambda) S(C-B)+S(B \cap C) .
$$

\section{REFERENCES}

1. V. Bergström, Ein neuer Beweis eines Satzes von E. Steinitz, Abh. Math. Sem. Univ. Hamburg. 8 (1931), 148-154.

2. —_ Zwei Sätze über ebene Vectorpolygone, Abh. Math. Sem. Univ. Hamburg. 8 (1931), 206-214.

3. D. J. Brown, Existence of a competitive equilibrium in a nonstandard exchange economy, Cowles Foundation Discussion Paper No. 342, Cowles Foundation for Research in Economics, Yale University, New Haven, Conn.

4. I. Damsteeg and I. Halperin, The Steinitz-Gross theorem on sums of vectors, Trans. Roy. Soc. Canada (3) 44 (1950), 31-35. MR 12, 419.

5. A. P. Robertson and J. F. C. Kingman, On a theorem of Lyapunov, J. London Math. Soc. 43 (1968), 347-351. MR 37 \#367.

6. A. Robinson, Non-standard analysis, North-Holland, Amsterdam, 1966. MR 34 $\# 5680$.

7. E. Steinitz, Bedingt konvergente Reihen und konvexe Systeme, J. Reine Angew. Math. 143 (1913), 128-175.

Department of Mathematics, Yale University, New Haven, Connecticut 06520

Current address: Department of Mathematics, University of Illinois, Urbana, Illinois 61801 